\title{
EDITORIAL
}

\section{The NDPK/NME superfamily: state of the art}

\author{
Mathieu Boissan ${ }^{1,2}$, Uwe Schlattner ${ }^{3}$ and Marie-Lise Lacombe ${ }^{1}$
}

Nucleoside diphosphate kinases (NDPK) are nucleotide metabolism enzymes encoded by NME genes (also called NM23). Given the fact that not all NME-encoded proteins are catalytically active NDPKs and that NM23 generally refers to clinical studies on metastasis, we use here NME/NDPK to denote the proteins. Since their discovery in the 1950's, NMEs/NDPKs have been shown to be involved in multiple physiological and pathological cellular processes, but the molecular mechanisms have not been fully determined. Recent progress in elucidating these underlying mechanisms has been presented by experts in the field at the 10th International Congress on the NDPK/NME/AWD protein family in October 2016 in Dubrovnik, Croatia, and is summarized in review articles or original research in this and an upcoming issue of Laboratory Investigation. Within this editorial, we discuss three major cellular processes that involve members of the multi-functional NME/NDPK family: (i) cancer and metastasis dissemination, (ii) membrane remodeling and nucleotide channeling, and iii) protein histidine phosphorylation.

Laboratory Investigation (2018) 98, 164-174; doi:10.1038/labinvest.2017.137

N ucleoside diphosphate kinases (NDPK) are nucleotide metabolism enzymes encoded by $N M E$ (also named NM23) genes. NDPKs are ubiquitous enzymes catalyzing the transfer of a phosphate from nucleoside triphosphates (NTPs) to nucleoside diphosphates (NDPs), in particular GDP, by a ping-pong mechanism involving the formation of a phosphohistidine intermediate. ${ }^{1-3}$ In this reaction, the main donor of phosphate is ATP, mainly provided by the mitochondrial oxidative phosphorylation, since its intracellular concentration is much higher than that of any other nucleoside triphosphate. ${ }^{4}$ In mammals, 10 genes have been identified to be a part of the NME/NDPK family. ${ }^{5,6}$ They encode

Sorbonne Universités, UPMC Univ Paris 06, INSERM, UMR S 938, Saint-Antoine Research Center, Paris, France:

${ }^{2}$ AP-HP, Hospital Tenon, Service de Biochimie et Hormonologie, Paris, France and ${ }^{3}$ Univ. Grenoble Alpes, INSERM, Laboratory of Fundamental and Applied Bioenergetics and SFR Environmental and Systems Biology, Grenoble „France Correspondence: E-mail: mathieu. boissan@inserm.fr proteins with one or two NDPK domains or a truncated NDPK domain, eventually associated with other domains whose functions remain largely unknown. Phylogenetic analysis shows that the NME genes are divided into two different groups. ${ }^{5,7}$ The group I genes encode proteins (NME1 to NME4) sharing 58 to $88 \%$ identity with each other. The four proteins NME1, NME2, NME3, and NME4 are ubiquitous and possess NDPK catalytic activity. They are mainly localized in the cytosol and at the plasma membrane except the NME4 isoform, which possesses a specific mitochondrial targeting sequence directing it to mitochondria (Lacombe et al, upcoming issue ${ }^{8}$ ). NME1, NME2, NME3, and NME4 form hexamers as the catalytically active form (Ćetković et al, next issue $^{9}$ ). Group II is comprised of more divergent proteins sharing only 22 to $44 \%$ identity with group I enzymes and between each other. These proteins are found in ciliated structures except for NME6, which is ubiquitous. NME1 and NME2 are the most abundant by far, and also the most studied, particularly NME1.

Constitutive knockout (KO) mice have been generated and reported for NME1, NME2, NME1 +NME2, NME5, and NME7 genes. ${ }^{10}$ NME1 KO mice exhibit a normal overall phenotype, with only a mild global hypotrophy and impaired mammary gland development. ${ }^{11,12}$ NME2 KO mice are phenotypically normal, except a defect in $\mathrm{K}^{+}$ channel Kca3.1 activation and cytokine production in $\mathrm{T}$ cells. ${ }^{13}$ In striking contrast, the double $\mathrm{KO}$ mice for both NME1 and NME2 genes die at birth, probably due to major defects in erythroid cell maturation. ${ }^{14,15}$ NME5 and NME7 knockout mice harbor a phenotype suggestive of primary ciliary dyskinesia, which is consistent with expression of NME5 and NME7 in ciliated structures. ${ }^{16}$ Finally, knockdown of NME2 in zebrafish induces severe cardiac dysfunction. ${ }^{17-19}$ Given the existence of multiple NME genes encoding various NME/NDPK proteins with possibly redundant functions, future 
generation of conditional knock-out and knock-in models should help us to understand their specific functions in a particular tissue or developmental stage, or for a given pathological process.

Besides their known function in the control of intracellular nucleotide homeostasis, which was discovered in the 1950's, NDPK proteins have been shown to be involved in multiple physiological and pathological cellular processes, mostly without a clear knowledge of the underlying molecular mechanisms. Based on the reports made at the 10th International Congress on the NME/NDPK/ Awd proteins in October 2016 in Dubrovnik, Croatia, experts in the field have summarized their ideas and views in review articles or contributed novel original research to two special issues of Laboratory Investigation. Within this editorial, we will focus on three major topics that emphasize recent progress in the field: (i) cancer and metastasis dissemination, (ii) membrane remodeling and nucleotide channeling, and (iii) protein histidine phosphorylation.

\section{NME IN METASTASIS}

Metastasis is the main cause of death in cancer patients. The first step in metastasis is the conversion from in situ carcinoma into invasive cancer, as the basement membrane is breached and invasive migration of tumor cells occurs through stromal fibrillar type I collagen. The tumor cells then enter the circulation and exit to colonize a secondary organ and form a metastasis. ${ }^{20-23}$ The mechamisms of the switch from in situ to invasive carcinoma remain largely unknown, although membrane type 1-matrix metalloproteinase (MT1MMP) is involved in this process. ${ }^{24,25}$ The identification of NME1 as the first metastasis suppressor gene ${ }^{26}$ has opened the field of this new class of metastasis dissemination regulators $\left({ }^{27-30}\right.$; Ćetković et al, upcoming issue; ${ }^{31}$ Khan and Steeg, ${ }^{32}$ this issue). Unlike tumor suppressor genes, metastasis suppressors inhibit in vivo metastasis without globally inhibiting primary tumor growth. In several solid tumor types, an inverse association between NME1 expression and metastatic spread has been observed. Specifically, three major findings have verified the anti-metastatic activity of NME1. First, the overexpression of NME1 in several metastatic cell lines including melanoma, breast, colon, lung, liver, ovary, prostate, and oral carcinoma cell lines, reduced their metastatic potential in xenograft models. ${ }^{33-39}$ Second, the incidence of lung metastases is significantly increased in NME1 knockout mice prone to develop hepatocellular carcinoma. ${ }^{40}$ Third, the silencing of NME1, but not of the highly homolog NME2, by RNA interference confers a metastatic phenotype to several non-invasive human epithelial tumoral cell lines by altering cell-cell adhesion, migration, invasion, and signaling linked to cancer progression. ${ }^{41}$ Invalidation of NME1 induced the destabilization of E-cadherinmediated adherens junctions, and the nuclear translocation of $\beta$-catenin associated with the transactivation of the transcription factor TCF/ LEF-1, all these events are hallmarks of epithelialmesenchymal transition 'EMT'. NME1 silenced hepatic and colon tumor cells acquired the ability to invade Matrigel, which mimics the basement membrane, and native type I collagen, the major component of the stroma. Similar increased invasiveness in Matrigel due to NME1 silencing has also been reported for uveal melanoma, lung, and breast cancer cells. ${ }^{41-44}$ NME1 silencing induced the formation of invadopodia, which are specialized plasma membrane protrusions endowed with MT1-MMP-dependent matrix degrading activity. ${ }^{41}$ Thus, several mechanisms converge to promote matrix proteolysis upon NME1 silencing. Pro-invasive signaling pathways that are activated upon NME1 silencing included Rac1, MAPK/SAPK, Akt, and TCF/LEF-1mediated transcription. ${ }^{41}$ NME1 silencing promotes upregulation of several MMPs including MT1-MMP. Accordingly, pharmacological inhibitors targeting Rho-GTPases, Src, PI3K/Akt, MAPK/SAPK, and MT1-MMP abrogated the proinvasive signaling induced by NME1 deficiency. Thus, it appears that multiple mechanisms induce a strong invasive and motile phenotype in response to NME1 silencing, in agreement with the multifunctional properties of this metastasis suppressor protein. In conclusion, NME1 emerges as a major upstream regulator of the metastatic signaling cascade.

The molecular mechanisms involved in the control of metastatic potential by NME/NDPK proteins are largely unknown (Farkas et al, ${ }^{45}$ this issue). They could involve several known NME1 enzymatic activities (NDPK, histidine kinase, and 3'-5' exonuclease), protein-protein interactions, and/or downstream gene regulation properties. A granzyme A-activated DNAse (GAAD) activity involved in caspase-independent apoptosis was also reported for NME1. ${ }^{46,47}$ Accumulating evidence suggests that cytosolic NME/NDPKs NME1 but also often NME2, interact with and affect different components and regulators of the cytoskeleton, including actin-binding proteins, intermediate filaments, and cytoskeleton 
attachment structures (adherens junctions, desmosomes, and focal adhesions) in cells from a variety of organisms and tissues, and in the course of development, suggesting that this association is evolutionarily conserved and may serve an essential function. ${ }^{48-55}$ The interactions of NDPK with components of the cytoskeletal machinery are highly relevant, given the well-established role of the cytoskeleton in cell motility, a critical determinant of the metastasis process. NME1 has also been reported to bind proteins belonging to small and heterotrimeric G-proteins $\left({ }^{56-61}\right.$; and Filic ${ }^{62}$ upcoming issue; Abu-Taha et al, ${ }^{63}$ this issue), transcriptional complexes ( ${ }^{64-69}$; Sharma et al, ${ }^{70}$ Pandey and Robertson, ${ }^{71}$ and Puts et al, ${ }^{72}$ all in this issue), and components of signaling pathways of MAPK, ${ }^{73-75}$ TGF- $\beta^{76-78}$ and Notch, ${ }^{79}$ as well as other factors promoting invasion and metastasis $\left({ }^{80,81}\right.$; Ferrucci et al ${ }^{82}$ upcoming issue). Accordingly, the list of protein/protein interactions involving NME1 is huge and could yield new information about the anti-metastatic activity of NME1, and more generally about the metastatic process. The role of the highly homologous isoform NME2 in metastasis dissemination remains much less documented and controversial. ${ }^{83-87}$ Our most recent data demonstrate a major role of NME1, not of NME2, during the transition of breast carcinoma from in situ to invasive (Mathieu Boissan, unpublished data).

Numerous clinical studies reported a crucial role of NME1 in cancer metastasis; indeed, an inverse association between NME1 expression and the metastatic potential for human solid tumors of epithelial origin such as breast, liver, colorectal, ovarian, and lung carcinoma and for melanoma has been reported. ${ }^{88}$ However, the link between NME, cancer and metastasis is more complicated, because of the biphasic expression of NME in cancer and metastasis: (i) an overexpression of both NME1 and NME2 isoforms in most human solid tumors at early stages of tumoral development ${ }^{41,89}$ as well as murine tumors, ${ }^{40}$ and (ii) a specifically decreased expression of NME1 in the primary tumor correlated with metastatic spread. ${ }^{89,90}$ Interestingly, a loss of NME1 was observed at the invasive front of hepatic and colorectal tumors. ${ }^{41}$ If for liver, breast, colon and lung carcinoma as well as for melanoma, the vast majority of studies reported an inverse correlation with metastasis and/or poor overall survival (Leonard et al, ${ }^{91}$ next issue), this is less marked in gastric and ovarian carcinoma, for which disparate results were found. ${ }^{88,92}$ In other types of cancers such as neuroblastoma, hematopoietic malignancies, and osteosarcoma, high tumoral expression was noted, which was most often correlated with poor outcome (Tan and Chang, ${ }^{93}$ this issue). The conflicting data described in the literature, might be due, at least partially, to the presence of the two closely related isoforms NME1 and NME2, which are most often not discriminated by antibodies and probes; heterogeneous expression in the primary tumors; and/or the criteria used to evaluate and grade NME1 expression in human clinical samples.

An important question little documented in the literature pertains to how NME1 is lost during invasive stages. Three mechanisms have been proposed: First, downregulation of NME1 expression may be the result of increased methylation of $\mathrm{CpG}$ islands mostly found around the transcription start site of the NME1 promoter as DNA methylation inhibitors can increase NME1 expression in tumor cells. ${ }^{94}$ Second, SWI/SNF chromatin remodelers-associated PRMT5 (protein arginine methyltransferase 5) is directly involved in transcriptional repression of NME1. ${ }^{95}$ Third, NME1 could be regulated at the protein level by (i) lysosomal cysteine cathepsins, proteases with known roles in invasion and metastasis, which directly cleave and degrade NME $1,{ }^{96}$ and (ii) the E3 ubiquitin ligase SCF-FBXO4, which interacts with NME1 to mediate its polyubiquitination and subsequent proteosomal degradation..$^{97}$ Finally, a field worth investigation in regard to NME1 tumoral expression is the potential role of the tumoral stroma, in particular, cancer-associated fibroblasts $(\mathrm{CAF})$, which have been shown to promote cancer invasion and migration, and metastasis in many different models. ${ }^{98}$ Taken together, these data strongly suggest that re-expression of NME1 in invasive cancer cells could constitute a promising strategy for anti-metastatic therapy. ${ }^{99,100}$

\section{NME IN MEMBRANE REMODELING AND NUCLEOTIDE CHANNELING}

Studies using several model systems, including Drosophila melanogaster, Caenorhabditis elegans, and mouse, as well as human, have demonstrated a fundamental role of the NME/NDPK family in endocytosis, intracellular trafficking, and nucleotide channeling (Figure 1). The starting point was a report in 2001 by neurobiologists, demonstrating a genetic and functional interaction between Awd and Shibire, the NME/NDPK and dynamin homologs, respectively, in D. melanogaster. ${ }^{101}$ Mutations in Awd (the corresponding protein Awd sharing 78\% aminoacid identity with the human NME1 and NME2 
isoforms) enhance paralysis of the Shibire mutant, a temperature-sensitive mutant of dynamin that blocks its function, resulting from defects in endocytosis-mediated neurotransmitter uptake at synaptic junctions. ${ }^{101}$ Remarkably, in a genetic screen to identify mutations that modify this phenotype, only Awd mutations were found, indicating that the functional relationship between Shibire and Awd is highly specific. More recent work in Drosophila epithelial cells, such as tracheal cells and border cells, confirmed the link between Shibire and Awd for internalization of growth factor receptor homologs for FGF and PDGF/ VEGF. ${ }^{102,103}$ Awd-dependent endocytosis also contributes to ensure epithelial integrity of follicular cells in the egg chamber by modulating the levels of adherens junction components. ${ }^{104} \mathrm{~A}$ number of further studies suggest links between NME and dynamin. The endocytic function of VHL (Von Hippel-Lindau) protein, a tumor suppressor that serves as a negative regulator of hypoxia-inducible factor-alpha subunits, regulates surface localization of FGF (fibroblast growth factor) receptor 1 via NME1, ${ }^{105}$ and ARF6-GTP, the active form of ARF6, interacts with and recruits NME1 to facilitate dynamin-mediated endocytosis. ${ }^{58} \mathrm{~A}$ novel genetic interaction was also found between Dyn-1 and NDK-1, the homologs of dynamin and NME/NDPK, respectively, in the nematode C. elegans during engulfment of apoptotic corpses. ${ }^{106}$ This process requires reorganization of cytoskeleton and membrane remodeling to extend the cell surface of the engulfing cell. Indeed, Dyn-1 and NDK-1 loss-offunction mutant embryos show phenotypic similarities, ie an accumulation of apoptotic cell corpses, and Dyn-1:NDK-1 double mutants are lethal. Moreover, in a genome-wide RNAi screen for genes involved in membrane trafficking, knockdown of NDK-1 caused failure in receptormediated endocytosis. ${ }^{107}$ In mammals, knockdown of NME1/NME2 impairs dynamin-mediated endocytosis of surface receptors such as the transferrin and EGF receptors, as well as of IL-2 receptor $\beta$ subunit in human and monkey cells; ${ }^{108}$ however, knockdown appears not to affect recycling of receptors since recycling of the transferrin receptor from endosomes to the plasma membrane is not altered. Taken together, these data clearly indicate that dynamin and NME/ NDPKs are close functional partners involved in membrane remodeling and trafficking across different species. ${ }^{109}$

Dynamin superfamily proteins are unique molecular motors that use GTP instead of ATP.
They have a very low affinity for GTP and a high basal rate of GTP hydrolysis. Polymerization into helical structures around the membrane neck of clathrin-coated pits (CCP) in vivo stimulates their GTPase activity. Accordingly, dynamin activity should be very sensitive to variations in the GTP level. ${ }^{108,110,111}$ This raises the hypothesis that NDPK activity of NME/NDPK proteins could directly affect the function of dynamin superfamily proteins through spatiotemporally controlled GTP production and availability, to meet elevated GTP turnover rates of dynamins. In support, loss of NME1/NME2 inhibits dynamin-mediated endocytosis and phenocopies the dynamin-null phenotype $^{108,112}$ ie, the augmentation of the density of CCPs and the tubular elongation of CCPs connected to the plasma membrane in comparison to controls. Thus, in the absence of NME1 and NME2 proteins, CCPs form properly but fail to detach from the plasma membrane, indicating a role for these NDPKs in dynaminmediated membrane fission at the CCPs. Local delivery of NME/NDPK-generated GTP to dynamins is further supported by the following findings: ${ }^{108,113}$ (i) NME1/NME2 colocalize at CCPs with the AP-2 complex, a major component involved in clathrin-mediated endocytosis, and dynamin, and they interact physically with dynamin, (ii) NME1/NME2 are recruited to dynamin-coated tubules and stimulate GTPloading on dynamin, and (iii) NME1/NME2 trigger dynamin-mediated membrane fission in the presence of ATP and GDP. Thus, cytosolic NME1/ NME2 channel GTP to classical cytosolic dynamins at plasma membrane CCPs to power their GTPase activity during endocytosis.

Besides the functional link between the two cytosolic NDPKs (NME1 and NME2) and classical endocytic dynamins (dynamin-1, expressed at high levels specifically in neuronal tissues and involved in synaptic vesicle endocytosis and dynamin-2, ubiquitously expressed and involved in clathrinmediated endocytosis (CME) as well as in some clathrin-independent endocytic pathways), the GTP channeling mechanism from NME/NDPK toward dynamins has been also shown in another subcellular compartment, the mitochondria. Here NME4 and the dynamin-related GTPase OPA1 are co-localized in the intermembrane space, bound to the inner mitochondrial membrane. Knockdown of NME4, but not of NME1/NME2, induces alterations of the mitochondrial morphology including fragmented and swollen-blebby mitochondria, which is reminiscent of a defect of mitochondria fusion. ${ }^{108,114}$ Furthermore, NME4 


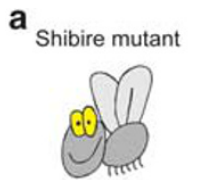

No paralysis at $19^{\circ} \mathrm{C}$

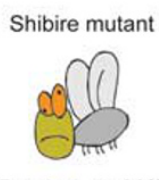

Paralysis at $29^{\circ} \mathrm{C}$
Shibire mutant + awd mutations

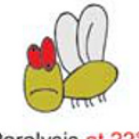

Paralysis at $22^{\circ} \mathrm{C}$

b
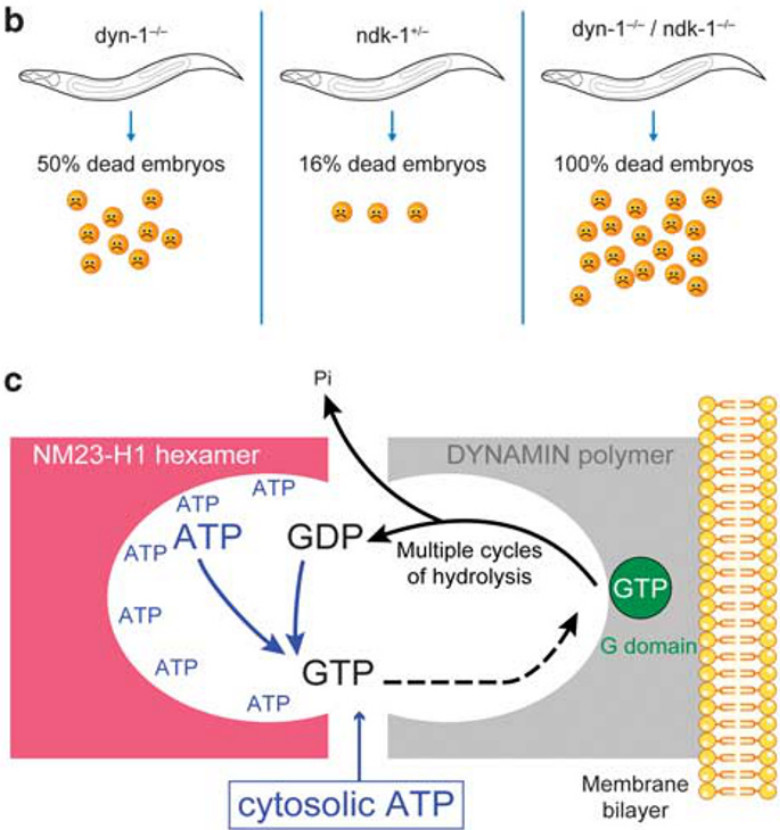

d

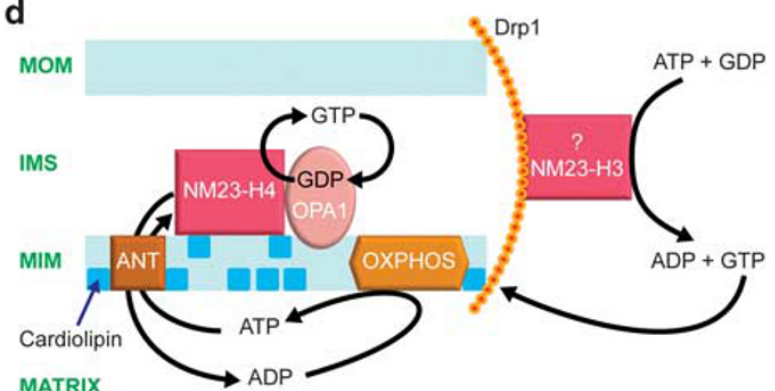

MATRIX

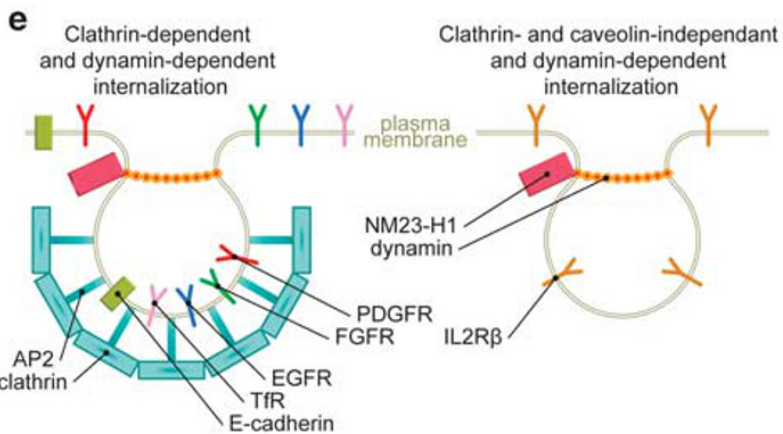

NDPK co-localizes and interacts with OPA1 at the inner membrane, ${ }^{115}$ and increases GTP-loading on OPA1, indicating a scheme in which NME4 provides a local GTP supply to OPA1 at the inner mitochondrial membrane. ${ }^{108}$ Taken together, the evidence reported above supports a model in which NMEs/NDPKs physically interact with dynamin superfamily proteins in the same subcellular compartment to maintain a high local concentration of GTP for dynamin's function in 
Figure 1 (a) Genetic interaction between NM23 and dynamin homologs in Drosophila. Left: a mutant of the dynamin homolog in Drosophila (shibire), which is temperature sensitive, present no paralysis at a permissive temperature, $19^{\circ} \mathrm{C}$. Middle: the shibire mutant present paralysis of the flies due to defects in endocytosis-mediated neurotransmitter uptake at a non-permissive temperature, $29^{\circ} \mathrm{C}$. Right: combination of mutations of awd, the homolog of NM23-H1/-H2 in Drosophila, and mutations of shibire induces paralysis at a lower temperature $22{ }^{\circ} \mathrm{C}$, indicating that mutations of NM23 make synapses more sensitive to dynamin mutations. (b) Genetic interaction between NM23 and dynamin homologs in C. elegans. In order to test whether NDPK/NDK-1 and DYN-1/Dynamin also interact in the worm genetically, ndk-1-/-;dyn-1-/- double mutants were generated. The viable thermosensitive ky51 allele of dyn-1 (dyn-1-/-) results in 50\% embryonic lethality at the restrictive temperature $\left(25^{\circ} \mathrm{C}\right)$. These embryos arrest development at a late embryonic stage due to endocytosis defects and display an accumulation of cell corpses. In the progeny of ndk-1+/- heterozygotes $16 \%$ of ndk-1-/-;dyn-1-/-homozygotes die as embryos showing the Dyn-1-like late embryonic lethality phenotype with persistent cell corpses. Double mutants at the restrictive temperature resulted in no viable progeny (100\% embryonic lethality). (c) NM23-H1 a GTP dealer for dynamin at clathrin-coated pits during endocytosis. Dynamin is recruited at the plasma membrane clathrin-coated pits, polymerizes into helix which strongly stimulates its GTPase activity inducing GTP hydrolysis; multiple cycles of GTP loading and hydrolysis are necessary for constriction and fission. To load very efficiently and locally the GTP on dynamin, NM23-H1 physically interacts with dynamin at clathrin-coated pits and uses the GDP coming from the GTP hydrolysis and the cytosolic ATP pool to produce GTP in a closed circuit. Finally, to increase the efficiency of the reaction, a hexamer of NM23-H1 interacts with dynamin, this hexamer containing six active sites that can function in concert, ensuring in a same time six catalytic reactions. (d) NM23-H4 controls mitochondrial fusion through a functional coupling with the dynamin-related GTPase OPA1. A hexamer of NM23-H4 binding to the mitochondrial inner membrane harbors NDPK activity for regeneration of NTP, mainly GTP, in the mitochondrial intermembrane space, and for direct and local GTP fueling (channeling-tunneling) of the dynamin-related protein OPA1. NM23-H4 also channels ADP via adenylate translocase (ANT) into the matrix space for stimulation of respiration and ATP regeneration through oxidative phosphorylation (OXPHOS). The localization of NM23-H3 at the mitochondrial surface where the dynamin-related pro-fission protein Drp1 acts could suggest that the NDPK NM23-H3 could assist Drp1 by GTP fueling during mitochondrial fission. MOM, mitochondrial outer membrane; IMS, intermembrane space; MIM, mitochondrial inner membrane; ANT, adenylate translocase; OXPHOS, oxidative phosphorylation. (e) NM23-H1 controls dynamin-mediated endocytosis. Left: NM23-H1 facilitates clathrin-dependent and dynamin-dependent internalization of E-cadherin, transferrin receptor (TfR), epidermal growth factor receptor (EGFR), fibroblast growth factor (FGFR), and platelet-derived growth factor receptor (PDGFR). Right: NM23-H1 facilitates clathrin- and caveolin-independent and dynamin-dependent internalization of the $\beta$ subunit of the interleukin 2 receptor (IL2R $\beta$ ).

membrane remodeling. Interestingly, another mode of action of NME4 is also related to its capacity to bind membranes. The NME4 hexamer enables intermembrane transfer of the mitochondrial phospholipid cardiolipin between inner and outer mitochondrial membrane, which can serve as a signal for mitophagy or apoptosis, depending of the cardiolipin oxidation state. The bioenergetics and lipid signaling functions seem to be mutually exclusive, making this protein a sort of bifunctional nanoswitch (115,116; Schlattner et al, ${ }^{117}$ this issue).

\section{NMEs AS PROTEIN HISTIDINE KINASES}

Besides their well-established role as NDP kinases, there is emerging evidence that NME/NDPK proteins act as protein histidine kinases in mammals (Attwood and Muimo ${ }^{118}$ and Muimo et al, ${ }^{119}$ upcoming issue). For NME2/NDPK-B, at least two substrates were identified and studied in more detail: the $\beta$ subunit of heterotrimeric $\mathrm{G}$ protein $^{120-122}$ and the potassium channel KCa3.1. ${ }^{123-125}$ In the first case, NME2 forms a complex with $\beta / \gamma$ dimers of heterotrimeric $\mathrm{G}$ proteins, in which a phospho-relay occurs from His 118 of NME2 to His 266 of the G $\beta$ subunit. This ultimately leads to the formation of GTP from
GDP and thus receptor-independent activation of the $\mathrm{G}$ protein. The second well-documented example is the NME2-mediated activation of the $\mathrm{K}^{+}$channel $\mathrm{KCa} 3.1$, inducing cytokine production in $\mathrm{CD}^{+}$cells. After stimulation of the $\mathrm{T}$ cell receptor TCR, the PI3 kinase-C2 $\beta$ subunit synthesizes phosphoinositol from which the phosphate is transferred on His118 of NME2. ${ }^{125}$ The kinase directly binds and activates KCa3.1 by transferring the phosphate from His 118 to His 358 of the channel. It has also been shown that the mammalian histidine phosphatase PGAM5 dephosphorylates NME2 on His118, thereby inhibiting phosphorylation and activation of KCa3.1. ${ }^{125}$ These findings reveal a novel regulatory mechanism for a mammalian potassium channel and T-cell activation, and highlight a unique feature of histidine versus serine/threonine and tyrosine as a regulatory phosphorylation site. ${ }^{126}$ However, exactly how bulky protein substrates can gain access to the active site of NME/NDPK proteins with the essential His118 situated in a cleft remains to be studied and explained by biochemical and crystallographic studies. More recently, anti-phosphohistidine antibodies have been obtained that will provide unique tools to 
scrutinize the role of histidine phosphorylation by NME/NDPK and dephosphorylation by phosphatases in various cellular processes (126,127; Makwana et al, ${ }^{128}$ next issue; Adam and Hunter, ${ }^{129}$ this issue).

\section{CONCLUDING REMARKS AND PERSPECTIVES}

Over the last three decades, extensive analyses of the NME/NDPK family started to reveal its multifaceted roles in cellular pathophysiology and the underlying molecular mechanisms. Beginning from a simple kinase activity, the field faces today the complexity of 10 different isoforms, all having specific features and often alternative, additional activities. The roles of these various isoforms undoubtedly require specific targeting and location within the cell. Most studies addressed the specific roles of NME1 and NME2, the two most abundantly expressed isoforms, with particular emphasis on the anti-metastasis function of NME1. Since Steeg's pioneering work on the antimetastasis potential of NME1, most studies confirmed its invasive and metastasis suppressor function in many different cellular and murine models using transfection experiments and knockout mouse strategies. However, translation of these findings to the clinics, eg, by modulating NME1 expression levels, is still lagging behind. Indeed, medroxyprogesterone acetate (MPA) has been previously identified as a potential candidate compound for clinical setting as NME1 expression increased in vivo in mice treated by MPA and contributed to the diminution of metastasis, but unfortunately it showed no therapeutic benefit in women with metastatic breast cancer. ${ }^{130,131}$ Thus, to date NME1 is not 'drug-able'. If the antimetastasis role of NME1 has been clearly reported in a large variety of tumor types, we do not know how its expression is regulated and in particular downregulated in the invasive component. Thus, it is of utmost importance to discover how it is modulated at the transcriptional, translational, and posttranslational level. Also, it would be of great interest to determine the role of the stromal microenvironment mainly composed of type I fibrillary collagen and cancer-associated fibroblasts (CAF), and its stiffness in the regulation of NME1 expression. Moreover, selective silencing of NME1 or NME2 does not alter the protein level of the other isoform, indicating that either protein does not interfere with the expression or stability of the other isoform. ${ }^{108}$ It is of great importance to remind that these isoforms show $88 \%$ identity at the protein level; sixteen of the eighteen different amino acids between NME1 and NME2 are located at the lateral surface of the hexamer, which could give rise to different interactions with other proteins and hence different cellular functions. NME1 and NME2 recombinant proteins form heterohexamers (Ioan Lascu, Marie-Lise Lacombe, unpublished data). If this is also the case in vivo, formation of such heterohexamers could introduce a supplementary level of cellular regulation. Indeed, NME1 can be co-immunoprecipitated with NME2 and vice versa in vivo (Mathieu Boissan, unpublished data), and thus the NME1/ NME2 ratio could be crucial for cellular functions of NME/NDPK.

The role of the NME/NDPK family in membrane remodeling and nucleotide channeling has become widely recognized as another feature of several family members, including at least NME1, NME2, NME4, and potentially NME3. ${ }^{108}$ Here the classical NDPK activity as the main source of GTP is extended to a direct GTP fueling of GTPdependent members of the dynamin superfamily through direct protein/protein interaction. This was shown in different species and model systems, including the fruit fly $D$. melanogaster, the nematode C. elegans and human, indicating an evolutionary conserved mechanism of membrane remodeling controlled by NME/NDPK proteins. GTP fueling to cytosolic dynamins (through cytosolic NME1 and NME2) affects endocytosis of cell surface receptors, while GTP fueling to mitochondrial dynamin-related OPA1 (through mitochondrial NME4 isoform) affects mitochondrial inner membrane fusion and cristae remodeling. The localization of NME3 at the outer mitochondrial membrane, where the dynamin-like protein DRP1 is recruited to mediate mitochondrial fission, suggests that NME3 might, likewise, assist DRP1 in this process. These observations provide a biochemical and thermodynamic explanation of why dynamin superfamily members are dependent on NDPKs. However, to fully validate this model, more comprehensive structural analyses of the interactions between NDPKs in their hexameric state and dynamin superfamily proteins in their polymeric state are needed. In addition, NME4 has an alternative signaling function. By binding to and transferring cardiolipin between inner and outer mitochondrial membrane in damaged mitochondria, it can trigger mitophagy or apoptosis. Recruitment of NME proteins to dynamin superfamily proteins as well as lipid transfer induced by the NME4 protein could suggest a general role of NME proteins in providing an interaction scaffold as well as nucleoside triphosphate channeling. ${ }^{113}$ It remains 
to be shown how these molecular mechanisms contribute to pathological states at the level of organisms, since the novel mitochondrial functions have potential for the development of interventions in various human pathologies.

Further, during the last decade, it became evident that certain NME/NDPK family members could act as a protein histidine kinase. In particular, NME2 has at least two defined substrates, the $\beta$ subunit of heterotrimeric $\mathrm{G}$ proteins and the intermediate conductance potassium channel KCa3.1. In all these proteins, the phosphorylation of a specific histidine residue is of regulatory importance for protein function, and NME2 seems to be the only known kinase able to catalyze this phosphorylation. There is apparently a direct transfer of the phosphoryl group between the phosphohistidine in the active site of the autophosphorylated NME2 and the histidine in the substrate protein. As mentioned above, the primary function of NME/NDPK is to catalyze phosphoryl transfer between NTPs and NDPs. Hence, how can this function be reconciled with phosphoryl transfer from NTPs to histidine on substrate proteins through NDPK activity is an important so far unresolved question. Specific anti-phosphohistidine antibodies will help to decipher the physiological relevance of histidine phosphorylation and more accurately the precise role of NME proteins in this process.

Finally, since more than one decade, it has been shown that some tumor cell lines overexpressing NME1 secrete this protein into the extracellular environment ( ${ }^{132}$; Romani et al, ${ }^{133}$ this issue; Khanim and Bunce, ${ }^{134}$ upcoming issue). Indeed, high concentrations of NME1 were found in the sera of patients with tumors overexpressing NME1. Tumor cells may secrete NME1 through unknown mechanisms, since there is no signal peptide sequence for secretion in the NME1 protein. In particular, the serum level of NME1 has been shown to be an important prognosis factor in acute myeloid leukemia. Secreted NME1 by tumor cells may affect tumor growth as an autocrine growth factor. For now, we need to understand how NME1 is secreted and how secreted NME1 affects tumor progression.

In conclusion, it is fascinating to observe how NME/NDPK proteins regulate complex cellular events from development, nucleotide channeling, and histidine phosphorylation to metastasis dissemination, indicating that these proteins are definitely not just 'housekeeping enzymes'. The mechanisms underlying these biological functions are now emerging from a body of working models, slowly but steadily validated by experimental studies, including several high-profile publications. Further biochemical, developmental, and molecular biology studies, targeting in particular the specific subcellular compartmentalization of NME/NDPK proteins, mutational assays, and finally animal models will undoubtedly further disentangle and validate NME/NDPK functions in human health and disease.

\section{DISCLOSURE/CONFLICT OF INTEREST}

The authors declare no conflict of interest.

1. Cleland WW. The kinetics of enzyme-catalyzed reactions with two or more substrates or products. I. Nomenclature and rate equations. Biochim Biophys Acta 1963;67: 104-137.

2. Norman AW, Wedding RT, Black MK. Detection of phosphohistidine in nucleoside diphosphokinase isolated from Jerusalem artichoke mitochondria. Biochem Biophys Res Commun 1965;20:703-709.

3. Lascu I, Gonin P. The catalytic mechanism of nucleoside diphosphate kinases. J Bioenerg Biomembr 2000;32: 237-246.

4. Traut TW. Physiological concentrations of purines and pyrimidines. Mol Cell Biochem 1994;140:1-22.

5. Lacombe ML, Milon L, Munier A, et al. The human Nm23/nucleoside diphosphate kinases. J Bioenerg Biomembr 2000;32:247-258.

6. Boissan M, Dabernat S, Peuchant E, et al. The mammalian Nm23/NDPK family: from metastasis control to cilia movement. Mol Cell Biochem 2009;329:51-62.

7. Desvignes $T$, Pontarotti $P$, Fauvel $C$, et al. Nme protein family evolutionary history, a vertebrate perspective. BMC Evol Biol 2009;9:256.

8. Lacombe MLT-SM, Boissan M, Schlattner U. The mitochondrial nucleoside diphosphate kinase (NDPKD/NME4), a moonlighting protein for cell homeostasis. Lab Invest, in press.

9. Ćetković H, Bosnar MH, Perina D, Mikoč A, et al. Characterization of a Group I Nme protein of Capsaspora owczarzaki-a close unicellular relative of animals. Lab Invest 2018;98:306-316.

10. Boissan M, Lacombe ML. Learning about the functions of NME/NM23: lessons from knockout mice to silencing strategies. Naunyn Schmiedebergs Arch Pharmacol 2011;384:421-431.

11. Arnaud-Dabernat $S$, Bourbon PM, Dierich $A$, et al. Knockout mice as model systems for studying $\mathrm{nm} 23$ / NDP kinase gene functions. Application to the nm23M1 gene. J Bioenerg Biomembr 2003;35:19-30.

12. Deplagne C, Peuchant E, Moranvillier I, et al. The antimetastatic nm23-1 gene is needed for the final step of mammary duct maturation of the mouse nipple. PLoS ONE 2011;6:e18645.

13. Di L, Srivastava S, Zhdanova O, et al. Nucleoside diphosphate kinase B knock-out mice have impaired activation of the $\mathrm{K}+$ channel $\mathrm{KCa} 3.1$, resulting in defective $\mathrm{T}$ cell activation. J Biol Chem 2010;285:38765-38771.

14. Postel EH, Wohlman I, Zou X, et al. Targeted deletion of $\mathrm{Nm} 23$ /nucleoside diphosphate kinase $\mathrm{A}$ and $\mathrm{B}$ reveals their requirement for definitive erythropoiesis in the mouse embryo. Dev Dyn 2009;238:775-787.

15. Postel EH, Zou X, Notterman DA, et al. Double knockout Nme1/Nme2 mouse model suggests a critical role for NDP kinases in erythroid development. Mol Cell Biochem 2009;329:45-50. 
16. Vogel $P$, Read $R$, Hansen GM, et al. Situs inversus in Dpcd/Poll-/-, Nme7-/-, and Pkd1/1-/- mice. Vet Pathol 2010;47:120-131.

17. Hippe HJ, Wolf NM, Abu-Taha I, et al. The interaction of nucleoside diphosphate kinase B with Gbetagamma dimers controls heterotrimeric $G$ protein function. Proc Natl Acad Sci USA 2009;106:16269-16274.

18. Hippe HJ, Wolf NM, Abu-Taha HI, et al. Nucleoside diphosphate kinase $B$ is required for the formation of heterotrimeric $\mathrm{G}$ protein containing caveolae. Naunyn Schmiedebergs Arch Pharmacol 2011;384:461-472.

19. Hippe HJ, Abu-Taha I, Wolf NM, et al. Through scaffolding and catalytic actions nucleoside diphosphate kinase $B$ differentially regulates basal and betaadrenoceptor-stimulated CAMP synthesis. Cell Signal 2011;23:579-585.

20. Steeg PS. Tumor metastasis: mechanistic insights and clinical challenges. Nat Med 2006;12:895-904.

21. Valastyan S, Weinberg RA. Tumor metastasis: molecular insights and evolving paradigms. Cell 2011;147: 275-292.

22. Chaffer $\mathrm{CL}$, San Juan BP, Lim E, et al. EMT, cell plasticity and metastasis. Cancer Metastasis Rev 2016:35:645-654.

23. Lambert AW, Pattabiraman DR, Weinberg RA. Emerging biological principles of metastasis. Cell 2017;168: 670-691.

24. Lodillinsky C, Infante E, Guichard A, et al. p63/MT1-MMP axis is required for in situ to invasive transition in basallike breast cancer. Oncogene 2016;35:344-357.

25. Castro-Castro A, Marchesin V, Monteiro P, et al. Cellular and molecular mechanisms of MT1-MMP-dependent cancer cell invasion. Annu Rev Cell Dev Biol 2016;32: 555-576.

26. Steeg PS, Bevilacqua G, Kopper L, et al. Evidence for a novel gene associated with low tumor metastatic potential. J Natl Cancer Inst 1988;80:200-204.

27. Steeg PS. Metastasis suppressors alter the signal transduction of cancer cells. Nat Rev Cancer 2003;3:55-63.

28. Steeg PS, Ouatas T, Halverson D, et al. Metastasis suppressor genes: basic biology and potential clinical use. Clin Breast Cancer 2003;4:51-62.

29. Rinker-Schaeffer CW, O'Keefe JP, Welch DR, et al. Metastasis suppressor proteins: discovery, molecular mechanisms, and clinical application. Clin Cancer Res 2006;12:3882-3889.

30. Smith SC, Theodorescu D. Learning therapeutic lessons from metastasis suppressor proteins. Nat Rev Cancer 2009;9:253-264.

31. Ćetković HRM, Harcet M, Bosnar MH. A survey of metastasis suppressors in Metazoa. Lab Invest, in press.

32. Khan I, Steeg PS. Metastasis suppressors: functional pathways. Lab Invest 2018;98:198-210.

33. Leone A, Flatow U, King CR, et al. Reduced tumor incidence, metastatic potential, and cytokine responsiveness of nm23-transfected melanoma cells. Cell 1991;65:25-35.

34. Leone A, Flatow U, VanHoutte $\mathrm{K}$, et al. Transfection of human $\mathrm{nm} 23-\mathrm{H} 1$ into the human MDA-MB-435 breast carcinoma cell line: effects on tumor metastatic potential, colonization and enzymatic activity. Oncogene 1993;8:2325-2333.

35. Palmieri D, Horak CE, Lee JH, et al. Translational approaches using metastasis suppressor genes. J Bioenerg Biomembr 2006;38:151-161.

36. Horak CE, Mendoza A, Vega-Valle $\mathrm{E}$, et al. Nm23-H1 suppresses metastasis by inhibiting expression of the lysophosphatidic acid receptor EDG2. Cancer Res 2007;67:11751-11759.

37. Steeg PS. Heterogeneity of drug target expression among metastatic lesions: lessons from a breast cancer autopsy program. Clin Cancer Res 2008;14:3643-3645.

38. Jarrett SG, Novak M, Dabernat S, et al. Metastasis suppressor NM23-H1 promotes repair of UV-induced
DNA damage and suppresses UV-induced melanomagenesis. Cancer Res 2012;72:133-143.

39. Jarrett SG, Novak M, Harris $N$, et al. NM23 deficiency promotes metastasis in a UV radiation-induced mouse model of human melanoma. Clin Exp Metastasis 2013;30:25-36.

40. Boissan $M$, Wendum $D$, Arnaud-Dabernat $S$, et al. Increased lung metastasis in transgenic NM23-Null/ SV40 mice with hepatocellular carcinoma. J Natl Cancer Inst 2005;97:836-845.

41. Boissan M, De Wever O, Lizarraga F, et al. Implication of metastasis suppressor NM23-H1 in maintaining adherens junctions and limiting the invasive potential of human cancer cells. Cancer Res 2010;70:7710-7722.

42. Bakalian S, Marshall JC, Faingold D, et al. Expression of $\mathrm{nm} 23-\mathrm{H} 1$ in uveal melanoma. Melanoma Res 2007;17: 284-290.

43. Duran MC, Vega F, Moreno-Bueno G, et al. Characterization of tumoral markers correlated with ErB2 (HER2/ $\mathrm{Neu}$ ) overexpression and metastasis in breast cancer. Proteomics Clin Appl 2008;2:1313-1326.

44. Ma W, Chen J, Xue X, et al. Alteration in gene expression profile and biological behavior in human lung cancer cell line NL9980 by nm23-H1 gene silencing. Biochem Biophys Res Commun 2008;371:425-430.

45. Farkas Z, Fancsalszky L, Saskői É, et al. The dosagedependent effect exerted by the NM23-H1/H2 homolog NDK-1 on distal tip cell migration in C. elegans. Lab Invest 2018;98:182-189.

46. Fan Z, Beresford PJ, Oh DY, et al. Tumor suppressor NM23-H1 is a granzyme A-activated DNase during CTLmediated apoptosis, and the nucleosome assembly protein SET is its inhibitor. Cell 2003;112:659-672.

47. Chowdhury D, Beresford PJ, Zhu P, et al. The exonuclease TREX1 is in the SET complex and acts in concert with NM23-H1 to degrade DNA during granzyme A-mediated cell death. Mol Cell 2006;23:133-142.

48. Lombardi D, Sacchi A, D'Agostino G, et al. The association of the Nm23-M1 protein and beta-tubulin correlates with cell differentiation. Exp Cell Res 1995;217:267-271.

49. Pinon VP, Millot G, Munier A, et al. Cytoskeletal association of the $A$ and $B$ nucleoside diphosphate kinases of interphasic but not mitotic human carcinoma cell lines: specific nuclear localization of the B subunit. Exp Cell Res 1999;246:355-367.

50. Otero AS. NM23/nucleoside diphosphate kinase and signal transduction. J Bioenerg Biomembr 2000;32:269-275.

51. Roymans D, Willems R, Vissenberg K, et al. Nucleoside diphosphate kinase beta (Nm23-R1/NDPKbeta) is associated with intermediate filaments and becomes upregulated upon CAMP-induced differentiation of rat C6 glioma. Exp Cell Res 2000;261:127-138.

52. Fournier HN, Dupe-Manet S, Bouvard D, et al. Integrin cytoplasmic domain-associated protein 1alpha (ICAP-1alpha ) interacts directly with the metastasis suppressor $\mathrm{nm} 23-\mathrm{H} 2$, and both proteins are targeted to newly formed cell adhesion sites upon integrin engagement. J Biol Chem 2002;277:20895-20902.

53. Aktary Z, Chapman K, Lam L, et al. Plakoglobin interacts with and increases the protein levels of metastasis suppressor $\mathrm{Nm} 23-\mathrm{H} 2$ and regulates the expression of Nm23-H1. Oncogene 2010;29:2118-2129.

54. Marino N, Marshall JC, Collins JW, et al. Nm23-h1 binds to gelsolin and inactivates its actin-severing capacity to promote tumor cell motility and metastasis. Cancer Res 2013;73:5949-5962.

55. Moreno V, Gonzalo P, Gomez-Escudero J, et al. An EMMPRIN-gamma-catenin-Nm23 complex drives ATP production and actomyosin contractility at endothelial junctions. J Cell Sci 2014;127:3768-3781.

56. Zhu J, Tseng YH, Kantor JD, et al. Interaction of the Rasrelated protein associated with diabetes rad and the 
putative tumor metastasis suppressor NM23 provides a novel mechanism of GTPase regulation. Proc Natl Acad Sci USA 1999;96:14911-14918.

57. Otsuki Y, Tanaka M, Yoshii S, et al. Tumor metastasis suppressor $\mathrm{nm} 23 \mathrm{H} 1$ regulates Rac1 GTPase by interaction with Tiam1. Proc Natl Acad Sci USA 2001;98: 4385-4390.

58. Palacios F, Schweitzer JK, Boshans RL, et al. ARF6-GTP recruits Nm23-H1 to facilitate dynamin-mediated endocytosis during adherens junctions disassembly. Nat Cell Biol 2002;4:929-936.

59. Iwashita S, Fujii M, Mukai $\mathrm{H}$, et al. Lbc proto-oncogene product binds to and could be negatively regulated by metastasis suppressor nm23-H2. Biochem Biophys Res Commun 2004;320:1063-1068.

60. Murakami M, Meneses PI, Knight JS, et al. Nm23-H1 modulates the activity of the guanine exchange factor Dbl-1. Int J Cancer 2008;123:500-510.

61. Murakami M, Meneses PI, Lan K, et al. The suppressor of metastasis Nm23-H1 interacts with the Cdc42 Rho family member and the pleckstrin homology domain of oncoprotein Dbl-1 to suppress cell migration. Cancer Biol Ther 2008;7:677-688.

62. Filić $V$, Marinović $M$, Šoštar $M$, et al. Modulation of small GTPase activity by NME proteins. Lab Invest, in press.

63. Abu-Taha $\mathrm{IH}$, Heijman J, Feng $Y$, et al. Regulation of heterotrimeric G-protein signaling by NDPK/NME proteins and caveolins: an update. Lab Invest 2018;98:190-197.

64. Subramanian C, Cotter 2nd MA , Robertson ES Epstein-Barr virus nuclear protein EBNA-3C interacts with the human metastatic suppressor Nm23-H1: a molecular link to cancer metastasis. Nat Med 2001;7:350-355.

65. Murakami M, Lan K, Subramanian C, et al. Epstein-Barr virus nuclear antigen 1 interacts with $\mathrm{Nm} 23-\mathrm{H} 1$ in lymphoblastoid cell lines and inhibits its ability to suppress cell migration. J Virol 2005;79:1559-1568.

66. Kaul R, Murakami M, Choudhuri T, et al. Epstein-Barr virus latent nuclear antigens can induce metastasis in a nude mouse model. J Virol 2007;81:10352-10361.

67. Kaul R, Murakami M, Lan K, et al. EBNA3C can modulate the activities of the transcription factor Necdin in association with metastasis suppressor protein Nm23H1. J Virol 2009:83:4871-4883.

68. Li $\mathrm{Y}, \mathrm{Nie} \mathrm{CJ}, \mathrm{Hu} \mathrm{L}$, et al. Characterization of a novel mechanism of genomic instability involving the SEI1/ SET/NM23H1 pathway in esophageal cancers. Cancer Res 2010;70:5695-5705.

69. Hung $\mathrm{CY}$, Wang YC, Chuang JY, et al. Nm23-H1stabilized hnRNPA2/B1 promotes internal ribosomal entry site (IRES)-mediated translation of Sp1 in the lung cancer progression. Sci Rep 2017;7:9166.

70. Sharma S, Sengupta A, Chowdhury S. NM23/NDPK proteins in transcription regulatory functions and chromatin modulation: emerging trends. Lab Invest 2018;98:175-181.

71. Pandey S, Robertson ES. Oncogenic Epstein-Barr virus recruits $\mathrm{Nm} 23-\mathrm{H} 1$ to regulate chromatin modifiers. Lab Invest 2018;98:258-268.

72. Puts GS, Leonard MK, Pamidimukkala NV, et al. Nuclear functions of NME proteins. Lab Invest 2018;98:211-218.

73. Hartsough MT, Morrison DK, Salerno M, et al. Nm23-H1 metastasis suppressor phosphorylation of kinase suppressor of Ras via a histidine protein kinase pathway. J Biol Chem 2002;277:32389-32399.

74. Salerno $M$, Palmieri $D$, Bouadis $A$, et al. Nm23-H1 metastasis suppressor expression level influences the binding properties, stability, and function of the kinase suppressor of Ras1 (KSR1) Erk scaffold in breast carcinoma cells. Mol Cell Biol 2005;25:1379-1388.

75. Peuchant E, Bats ML, Moranvillier I, et al. Metastasis suppressor NM23 limits oxidative stress in mammals by preventing activation of stress-activated protein
kinases/JNKs through its nucleoside diphosphate kinase activity. FASEB J 2017;31:1531-1546.

76. Hsu S, Huang F, Wang $L$, et al. The role of $n m 23$ in transforming growth factor beta 1-mediated adherence and growth arrest. Cell Growth Differ 1994;5:909-917.

77. Seong HA, Jung $\mathrm{H}, \mathrm{Ha} H$. NM23-H1 tumor suppressor physically interacts with serine-threonine kinase receptor-associated protein, a transforming growth factor-beta (TGF-beta) receptor-interacting protein, and negatively regulates TGF-beta signaling. J Biol Chem 2007;282:12075-12096.

78. Jung $\mathrm{H}$, Seong $\mathrm{HA}, \mathrm{Ha} H$. NM23-H1 tumor suppressor and its interacting partner STRAP activate p53 function. J Biol Chem 2007;282:35293-35307.

79. Ignesti $M$, Barraco $M$, Nallamothu $G$, et al. Notch signaling during development requires the function of awd, the Drosophila homolog of human metastasis suppressor gene Nm23. BMC Biol 2014;12:12.

80. Reymond A, Volorio S, Merla G, et al. Evidence for interaction between human PRUNE and $\mathrm{nm} 23-\mathrm{H} 1$ NDPKinase. Oncogene 1999;18:7244-7252.

81. D'Angelo A, Garzia L, Andre A, et al. Prune cAMP phosphodiesterase binds $\mathrm{nm} 23-\mathrm{H} 1$ and promotes cancer metastasis. Cancer Cell 2004;5:137-149.

82. Ferrucci V, Pennino FP, Siciliano $R$, et al. A competitive cell-permeable peptide (CPP) to impair the Nme-1 (NDPK-A) and Prune-1 interaction for therapeutic applications in cancer. Lab Invest, in press.

83. Thakur RK, Yadav VK, Kumar A, et al. Non-metastatic 2 (NME2)-mediated suppression of lung cancer metastasis involves transcriptional regulation of key cell adhesion factor vinculin. Nucleic Acids Res 2014;42:11589-11600.

84. Li Y, Tong $\mathrm{Y}$, Wong $\mathrm{YH}$. Regulatory functions of $\mathrm{Nm} 23$ $\mathrm{H} 2$ in tumorigenesis: insights from biochemical to clinical perspectives. Naunyn Schmiedebergs Arch Pharmacol 2015;388:243-256.

85. Kar A, Chowdhury S. Inhibition of telomerase activity by NME2: impact on metastasis suppression? Naunyn Schmiedebergs Arch Pharmacol 2015;388:235-241.

86. Tong Y, Yung LY, Wong YH. Metastasis suppressors $\mathrm{Nm} 23 \mathrm{H} 1$ and $\mathrm{Nm} 23 \mathrm{H} 2$ differentially regulate neoplastic transformation and tumorigenesis. Cancer Lett 2015; 361:207-217.

87. Liu YF, Yang A, Liu W, et al. NME2 reduces proliferation, migration and invasion of gastric cancer cells to limit metastasis. PLoS ONE 2015;10:e0115968.

88. Marino N, Nakayama J, Collins JW, et al. Insights into the biology and prevention of tumor metastasis provided by the Nm23 metastasis suppressor gene. Cancer Metastasis Rev 2012;31:593-603.

89. Martinez JA, Prevot S, Nordlinger B, et al. Overexpression of $\mathrm{nm} 23-\mathrm{H} 1$ and $\mathrm{nm} 23-\mathrm{H} 2$ genes in colorectal carcinomas and loss of $\mathrm{nm} 23-\mathrm{H} 1$ expression in advanced tumour stages. Gut 1995;37:712-720.

90. Boissan M, Lacombe ML. Nm23/NDP kinases in hepatocellular carcinoma. J Bioenerg Biomembr 2006;38:169-175.

91. Leonard MK, McCorkle JR, Snyder DE, et al. Identification of a gene expression signature associated with the metastasis suppressor function of NME1: prognostic value in human melanoma. Lab Invest 2018;98:329-340.

92. Hsu T, Steeg PS, Zollo $M$, et al. Progress on Nme (NDP kinase/Nm23/Awd) gene family-related functions derived from animal model systems: studies on development, cardiovascular disease, and cancer metastasis exemplified. Naunyn Schmiedebergs Arch Pharmacol 2015;388:109-117.

93. Tan $\mathrm{C}-\mathrm{Y}$, Chang CL. NDPKA is not just a metastasis suppressor-be aware of its metastasis-promoting role in neuroblastoma. Lab Invest 2018;98:219-227.

94. Hartsough MT, Clare SE, Mair M, et al. Elevation of breast carcinoma Nm23-H1 metastasis suppressor gene expression and reduced motility by DNA methylation inhibition. Cancer Res 2001;61:2320-2327. 
95. Pal $\mathrm{S}$, Vishwanath $\mathrm{SN}$, Erdjument-Bromage $\mathrm{H}$, et al. Human SWI/SNF-associated PRMT5 methylates histone H3 arginine 8 and negatively regulates expression of ST7 and NM23 tumor suppressor genes. Mol Cell Biol 2004;24:9630-9645.

96. Fiore LS, Ganguly SS, Sledziona J, et al. c-Abl and Arg induce cathepsin-mediated lysosomal degradation of the NM23-H1 metastasis suppressor in invasive cancer. Oncogene 2014;33:4508-4520.

97. Chen W, Xiong S, Li J, et al. The ubiquitin E3 ligase SCFFBXO24 recognizes deacetylated nucleoside diphosphate kinase $A$ to enhance its degradation. Mol Cell Biol 2015;35:1001-1013.

98. Orimo A, Weinberg RA. Heterogeneity of stromal fibroblasts in tumors. Cancer Biol Ther 2007;6:618-619.

99. Shoushtari AN, Szmulewitz RZ, Rinker-Schaeffer CW. Metastasis-suppressor genes in clinical practice: lost in translation? Nat Rev Clin Oncol 2011;8:333-342.

100. Carotenuto $\mathrm{M}$, de Antonellis $\mathrm{P}$, Chiarolla $\mathrm{CM}$, et al. A therapeutic approach to treat prostate cancer by targeting Nm23-H1/h-Prune interaction. Naunyn Schmiedebergs Arch Pharmacol 2015;388:257-269.

101. Krishnan KS, Rikhy $\mathrm{R}$, Rao $\mathrm{S}$, et al. Nucleoside diphosphate kinase, a source of GTP, is required for dynamin-dependent synaptic vesicle recycling. Neuron 2001;30:197-210.

102. Dammai V, Adryan B, Lavenburg KR, et al. Drosophila awd, the homolog of human $\mathrm{nm} 23$, regulates FGF receptor levels and functions synergistically with shi/ dynamin during tracheal development. Genes Dev 2003; 17:2812-2824.

103. Nallamothu G, Woolworth JA, Dammai V, et al. Awd, the homolog of metastasis suppressor gene Nm23, regulates Drosophila epithelial cell invasion. Mol Cell Biol 2008;28: 1964-1973.

104. Woolworth JA, Nallamothu G, Hsu T. The Drosophila metastasis suppressor gene $\mathrm{Nm} 23$ homolog, awd, regulates epithelial integrity during oogenesis. Mol Cell Biol 2009;29:4679-4690.

105. Hsu T, Adereth $\mathrm{Y}$, Kose $\mathrm{N}$, et al. Endocytic function of von Hippel-Lindau tumor suppressor protein regulates surface localization of fibroblast growth factor receptor 1 and cell motility. J Biol Chem 2006;281:12069-12080.

106. Fancsalszky L, Monostori E, Farkas Z, et al. NDK-1, the homolog of $\mathrm{NM} 23-\mathrm{H} 1 / \mathrm{H} 2$ regulates cell migration and apoptotic engulfment in C. elegans. PLoS ONE 2014;9: e92687.

107. Balklava Z, Pant S, Fares H, et al. Genome-wide analysis identifies a general requirement for polarity proteins in endocytic traffic. Nat Cell Biol 2007;9:1066-1073.

108. Boissan M, Montagnac G, Shen Q, et al. Membrane trafficking. Nucleoside diphosphate kinases fuel dynamin superfamily proteins with GTP for membrane remodeling. Science 2014;344:1510-1515.

109. Takacs-Vellai K, Vellai T, Farkas Z, et al. Nucleoside diphosphate kinases (NDPKs) in animal development. Cell Mol Life Sci 2015;72:1447-1462.

110. Morlot S, Roux A. Mechanics of dynamin-mediated membrane fission. Annu Rev Biophys 2013;42:629-649.

111. Antonny B, Burd C, De Camilli $P$, et al. Membrane fission by dynamin: what we know and what we need to know. EMBO J 2016;35:2270-2284.

112. Ferguson SM, Raimondi A, Paradise S, et al. Coordinated actions of actin and BAR proteins upstream of dynamin at endocytic clathrin-coated pits. Dev Cell 2009;17: 811-822.

113. Zala D, Schlattner U, Desvignes $T$, et al. The advantage of channeling nucleotides for very processive functions. F1000Res 2017;6:724.

114. Griparic $L$, van der Wel NN, Orozco IJ, et al. Loss of the intermembrane space protein Mgm1/OPA1 induces swelling and localized constrictions along the lengths of mitochondria. J Biol Chem 2004;279:18792-18798.
115. Schlattner U, Tokarska-Schlattner M, Ramirez S, et al. Dual function of mitochondrial $\mathrm{Nm} 23-\mathrm{H} 4$ protein in phosphotransfer and intermembrane lipid transfer: a cardiolipin-dependent switch. J Biol Chem 2013;288: 111-121.

116. Kagan VE, Jiang J, Huang $Z$, et al. NDPK-D (NM23-H4)mediated externalization of cardiolipin enables elimination of depolarized mitochondria by mitophagy. Cell Death Differ 2016;23:1140-1151.

117. Schlattner U, Tokarska-Schlattner M, Epand RM, et al. NME4/nucleoside diphosphate kinase D in cardiolipin signaling and mitophagy. Lab Invest 2018;98:228-232.

118. Attwood PV, Muimo R. The actions of NME1/NDPK-A and NME2/NDPK-B as protein kinases. Lab Invest 2018;98:285-292.

119. Muimo R, Alothaid HMM, Mehta A. NM23 proteins: innocent bystanders or local energy boosters for CFTR? Lab Invest 2018;98:274-284.

120. Cuello F, Schulze RA, Heemeyer F, et al. Activation of heterotrimeric $G$ proteins by a high energy phosphate transfer via nucleoside diphosphate kinase (NDPK) B and Gbeta subunits. Complex formation of NDPK B with Gbeta gamma dimers and phosphorylation of His-266 IN Gbeta. J Biol Chem 2003;278:7220-7226.

121. Lutz S, Hippe HJ, Niroomand F, et al. Nucleoside diphosphate kinase-mediated activation of heterotrimeric G proteins. Methods Enzymol 2004;390:403-418.

122. Hippe HJ, Luedde M, Lutz S, et al. Regulation of cardiac CAMP synthesis and contractility by nucleoside diphosphate kinase B/G protein beta gamma dimer complexes. Circ Res 2007;100:1191-1199.

123. Srivastava S, Li Z, Ko K, et al. Histidine phosphorylation of the potassium channel $\mathrm{KCa} 3.1$ by nucleoside diphosphate kinase $B$ is required for activation of KCa3.1 and CD4 T cells. Mol Cell 2006;24:665-675.

124. Srivastava S, Di L, Zhdanova O, et al. The class II phosphatidylinositol 3 kinase C2beta is required for the activation of the $\mathrm{K}+$ channel KCa3.1 and CD4 T-cells. Mol Biol Cell 2009;20:3783-3791.

125. Panda S, Srivastava S, Li Z, et al. Identification of PGAM5 as a Mammalian Protein Histidine Phosphatase that Plays a Central Role to Negatively Regulate CD4(+) T Cells. Mol Cell 2016;63:457-469.

126. Fuhs SR, Hunter T. pHisphorylation: the emergence of histidine phosphorylation as a reversible regulatory modification. Curr Opin Cell Biol 2017;45:8-16.

127. Fuhs SR, Meisenhelder J, Aslanian A, et al. Monoclonal 1and 3-Phosphohistidine Antibodies: New Tools to Study Histidine Phosphorylation. Cell 2015;162:198-210.

128. Makwana MV, Muimo R, Jackson RFW. Advances in development of new tools for the study of phosphohistidine. Lab Invest 2018;98:293-305.

129. Adam K, Hunter T. Histidine kinases and the missing phosphoproteome from prokaryotes to eukaryotes. Lab Invest 2018;98:233-247.

130. Palmieri D, Halverson DO, Ouatas T, et al. Medroxyprogesterone acetate elevation of $\mathrm{Nm} 23-\mathrm{H} 1$ metastasis suppressor expression in hormone receptor-negative breast cancer. J Natl Cancer Inst 2005;97:632-642.

131. Miller KD, Althouse SK, Nabell L, et al. A phase II study of medroxyprogesterone acetate in patients with hormone receptor negative metastatic breast cancer: translational breast cancer research consortium trial 007. Breast Cancer Res Treat 2014;148:99-106.

132. Okabe-Kado J, Kasukabe T. Physiological and pathological relevance of extracellular NM23/NDP kinases. J Bioenerg Biomembr 2003;35:89-93.

133. Romani $P$, Ignesti $M$, Gargiulo $G$, et al. Extracellular NME proteins: a player or a bystander? Lab Invest 2018;98: 248-257.

134. Bunce CM, Khanim FL, The 'known-knowns', and 'knownunknowns' of extracellular Nm23-H1/NDPK proteins. Lab Invest, in press. 